\title{
Some results about functions that share functions with their derivative of higher order
}

\author{
Jianming Qi ${ }^{1}$, Feng Lü ${ }^{2}$ and Wenjun Yuan ${ }^{3,4^{*}}$
}

${ }^{*}$ Correspondence: gzywj@tom.com
${ }^{3}$ School of Mathematics and
Information Science, Guangzhou
University, Guangzhou, 510006, P.R.
China
${ }^{4}$ Key Laboratory of Mathematics
and Interdisciplinary Sciences of
Guangdong Higher Education
Institutes, Guangzhou University,
Guangzhou, 510006, P.R. China
Full list of author information is
available at the end of the article

available at the end of the article

\begin{abstract}
In this paper, we investigate the growth of some functions that share functions with their derivative of higher order. The first main theorem is an improvement of the result obtained by Lü (Bull. Korean Math. Soc. 48: 951-957, 2011), two examples are given to show that the conclusion is sharp. The second main theorem is of estimating, more exactly, the order of an entire function sharing polynomial, which extends the related result of Lü, Xu and Chen (Arch. Math. 92: 593-601, 2009).
\end{abstract}

MSC: 30D35; 30D45

Keywords: complex differential equation; entire function; normal family; Nevanlinna theory

\section{Introduction and main results}

In this paper, a meromorphic function always means meromorphic in the whole complex plane. We assume that the reader is familiar with the basic notions of Nevanlinna theory. In the Nevanlinna theory, the order and the hyper-order of a meromorphic function are two important concepts. So, it is meaningful to discuss the properties of the order and the hyper-order for a meromorphic function. Let us recall the definitions of the order and the hyper-order of a meromorphic function $f$, which are respectively defined as (see [1])

$$
\begin{aligned}
& \rho(f)=\limsup _{r \rightarrow \infty} \frac{\log T(r, f)}{\log r}=\limsup _{r \rightarrow \infty} \frac{\log \log M(r, f)}{\log r}, \\
& \sigma(f)=\limsup _{r \rightarrow \infty} \frac{\log \log T(r, f)}{\log r}=\limsup _{r \rightarrow \infty} \frac{\log \log \log M(r, f)}{\log r} .
\end{aligned}
$$

In addition, we say that two meromorphic functions $f(z)$ and $g(z)$ share a finite value $a$ IM (ignoring multiplicities) when $f(z)-a$ and $g(z)-a$ have the same zeros. And we say that $f(z)$ and $g(z)$ share a finite value $a \mathrm{CM}$ (counting multiplicities) when $f(z)-a$ and $g(z)-a$ have the same zeros counting multiplicities. $\operatorname{deg} P(z)$ denotes the degree of the polynomial $P(z)$. If $R(z)=\frac{P_{1}(z)}{P_{2}(z)}$ is a rational function (where $P_{2}(z) \not \equiv 0$ and $P_{1}(z), P_{2}(z)$ are two coprime polynomials), then we indicate $\operatorname{deg} R(z)=\max \left\{\operatorname{deg} P_{1}(z), \operatorname{deg} P_{2}(z)\right\}$ to denote the degree of the rational function.

The subject on sharing values between entire functions and their derivatives was first studied by Rubel and Yang [2]. In 1977, they proved the result that if a nonconstant entire function $f$ and its first derivative $f^{\prime}$ share two distinct finite numbers $a, b \mathrm{CM}$, then $f \equiv f^{\prime}$. Since then, shared value problems have been studied by many authors and a number of profound results have been obtained (see, e.g., $[3,4]$ ).

() 2013 Qi et al.: licensee Springer. This is an Open Access article distributed under the terms of the Creative Commons Attribution License (http://creativecommons.org/licenses/by/2.0), which permits unrestricted use, distribution, and reproduction in any medium, provided the original work is properly cited. 
In 1982, Bank and Laine [5] investigated the complex oscillation theory of differential equations and obtained the following main result.

Theorem A Let $A(z)$ be a nonconstant polynomial of degree $n$, and let $f_{1}$ and $f_{2}$ be two linearly independent solutions of the equation $f^{\prime \prime}+A(z) f=0$. Then at least one of $f_{1}$ and $f_{2}$ has the property that the exponent of convergence of its zero-sequence is $\frac{n+2}{2}$.

Since then, to study properties of the exponent of convergence, the order and the hyperorder for the solutions of some differential equations becomes a hot topic and is discussed by many experts.

In 2008, Li and Gao [6] deduced the following result.

Theorem B Let $Q_{1}$ and $Q_{2}$ be two nonzero polynomials, and let $P$ be a polynomial. Iff is a nonconstant solution of the equation

$$
f^{(k)}-Q_{1}=e^{P}\left(f-Q_{2}\right),
$$

then $\sigma(f)=n$, where, and in the sequel, $n$ denotes the degree of $P$.

Recently, Lü [7] obtained the result.

Theorem C Let $f$ be a transcendental meromorphic function with finitely many poles, let $n \geq 2$ be an integer, and let $\alpha=P e^{Q}\left(\neq \alpha^{\prime}\right)$ be an entire function such that the order of $\alpha$ is less than that of $f$, where $P, Q$ are two polynomials. If $f^{n}$ and $\left(f^{n}\right)^{\prime}$ share $\alpha C M$, then $f=A e^{\frac{1}{n} z}$, where $A$ is a nonzero constant.

From Theorem C, we see that $f^{n}$ and $\left(f^{n}\right)^{\prime}$ share a function with finite order. So, it is natural to ask what will happen if they share functions with infinite order and also what will happen if $\left(f^{n}\right)^{\prime}$ is replaced by $\left(f^{n}\right)^{(k)}$. In this work, we discuss these problems and derive the following result.

Theorem 1.1 Let $f$ be a meromorphic function with finitely many poles, let $R$ be a rational function, $\gamma$ be an entire function. If all zeros of $f$ have multiplicity at least $k+1$ and

$$
f^{(k)}(z)=\alpha(z) \quad \Rightarrow \quad f(z)=\alpha(z)
$$

where $\alpha=\operatorname{Re}^{\gamma}$, then $\sigma(f) \leq \sigma(\alpha)=\rho(\gamma)$.

Remark 1 The following examples show that the conclusion $\sigma(f) \leq \rho(\gamma)$ is sharp.

Example 1 Let $f(z)=A e^{z}$, where $A$ is a nonzero constant. Let $\alpha(z)=e^{e^{z}+z^{2}}$. Noting that $f \equiv f^{(k)}$, we have

$$
f^{(k)}(z)=\alpha(z) \quad \Rightarrow \quad f(z)=\alpha(z) .
$$

Thus, $\sigma(f)=0<\sigma(\alpha)=1$. 
Example 2 Let $f(z)=z e^{z^{2}}+e^{z / 2}, \alpha(z)=\left(4 z^{2}-z+2\right) e^{z^{2}}$, and $\gamma(z)=z^{2}$. Then

$$
\frac{f-\alpha}{f^{\prime}-\alpha}=2 \text {. }
$$

Thus, $\sigma(f)=\rho(\gamma)=0$.

Remark 2 If $\gamma$ is a polynomial, then the above condition obviously holds.

Remark 3 In Theorem 1.1, if the order of $\gamma$ is zero, for example, $\gamma$ is a polynomial, then $\sigma(f)=0$.

In 2009, Lü, Xu and Chen [8] obtained the following result.

Theorem D Let $f(z)$ be a nonconstant meromorphic function with finitely many poles, and let $Q_{1}, Q_{2}\left(\not \equiv Q_{1}\right)$ be two polynomials. If

$$
f(z)=Q_{1}(z) \quad \Rightarrow \quad f^{\prime}(z)=Q_{1}(z) \text { and } f(z)=Q_{2}(z) \quad \Rightarrow \quad f^{\prime}(z)=Q_{2}(z),
$$

then $f(z)$ is of finite order.

In this paper, we get the following results which improve Theorem D.

Theorem 1.2 Let $f(z)$ be a nonconstant meromorphic function with finitely many poles, and let $Q_{1}, Q_{2}\left(\not \equiv Q_{1}\right)$ be two polynomials. If

$$
f(z)=Q_{1}(z) \quad \Rightarrow \quad f^{\prime}(z)=Q_{1}(z) \text { and } f(z)=Q_{2}(z) \quad \Rightarrow \quad f^{\prime}(z)=Q_{2}(z),
$$

then $\rho(f) \leq 2+2 \max \left\{\operatorname{deg} R_{1}(z), \operatorname{deg} R_{2}(z)\right\}$, where $R_{1}=\frac{Q_{2}-Q_{1}^{\prime}}{Q_{2}-Q_{1}}, R_{2}=\frac{Q_{1}-Q_{1}^{\prime}}{Q_{2}-Q_{1}}$ are rational functions.

Corollary 1.3 Let $f(z)$ be a nonconstant entire function and let $Q_{1}, Q_{2}\left(\not \equiv Q_{1}\right)$ be two polynomials. If $f(z)=Q_{1}(z) \Rightarrow f^{\prime}(z)=Q_{1}(z)$ and $f(z)=Q_{2}(z) \Rightarrow f^{\prime}(z)=Q_{2}(z)$, then $\rho(f) \leq$ $1+\max \left\{\operatorname{deg} R_{1}(z), \operatorname{deg} R_{2}(z)\right\}$, where $R_{1}=\frac{Q_{2}-Q_{1}^{\prime}}{Q_{2}-Q_{1}}, R_{2}=\frac{Q_{1}-Q_{1}^{\prime}}{Q_{2}-Q_{1}}$ are rational functions.

Very recently, Li, Gao and Zhang [9] proved the following result.

Theorem $\mathbf{E}$ Let $f$ be a nonconstant entire function. If $f$ and $f^{\prime}$ share the value $1 C M$, and if $N\left(r, \frac{1}{f^{\prime}}\right)<\alpha T(r, f)$, where $\alpha \in\left[0, \frac{1}{4}\right]$, then $f^{\prime}-1=c(f-1)$ for some nonzero constant $c$.

By the same method of Li, Gao and Zhang [9], we also consider the $k$ th derivative and improve the above result as follows.

Theorem 1.4 Let $f$ be a nonconstant entire function, and let $k$ be a positive integer. Iff and $f^{(k)}$ share the value $1 C M$ and if $N\left(r, \frac{1}{f^{(k)}}\right)<\alpha T(r, f)$, where $\alpha \in\left[0, \frac{1}{4}\right)$, then $f^{(k)}-1=c(f-1)$ for some nonzero constant c. 


\section{Some lemmas}

In order to prove our theorems, we need the following lemmas.

Lemma 2.1 [3] Let $\mathcal{F}$ be a family of meromorphic functions in the unit disc $\Delta$ with the property that for each $f(z) \in \mathcal{F}$, all zeros of $f(z)$ have multiplicity at least $k+1$. If $k$ is a positive integer and $a_{n} \rightarrow a,|a|<1$ and $f_{n}^{\sharp}\left(a_{n}\right) \rightarrow \infty$, there exist

1. a subsequence of functions $f_{n} \in \mathcal{F}$ (also denoted by $f_{n}$ );

2. a sequence of complex numbers $z_{n} \rightarrow z_{0},\left|z_{0}\right|<1$;

3. a positive sequence $\rho_{n} \rightarrow 0$;

4. $\frac{f_{n}\left(z_{n}+\rho_{n} \xi\right)}{\rho_{n}^{k}}=g_{n}(\xi) \rightarrow g(\xi)$, here $g$ is a nonconstant meromorphic (entire) function satisfying $g^{\sharp}(\xi) \leq g^{\sharp}(0)=k+1$ and

$$
\rho_{n} \leq \frac{M}{\sqrt[k+1]{f_{n}^{\sharp}\left(a_{n}\right)}},
$$

here $M, n$ are respective positive numbers.

With a similar method to that in [3, Lemma 2], we obtain the following Lemma 2.3, which plays an important part in the proof of Theorem 1.1. For the sake of convenience, the detailed proof will be given after Lemma 2.3.

Lemma 2.2 Let $f$ be a meromorphic function of hyper-order $\sigma(f)>0$. Then, for any $\epsilon>0$, there exists a sequence $z_{n} \rightarrow \infty$ such that $f^{\sharp}\left(z_{n}\right)>e^{\left|z_{n}\right|^{\sigma(f)-\epsilon}}$ if $n$ is large enough.

Proof On the contrary, there exist $\epsilon>0$ and $R>0$ such that $\epsilon<\sigma(f)$ and for all $z,|z| \geq R$ satisfying $f^{\sharp}(z) \leq e^{|z|^{\sigma(f)-\epsilon}}$. Thus,

$$
\begin{aligned}
S(r, f) & =\frac{1}{\pi} \iint_{|z|<r} f^{\sharp}(z)^{2} d \sigma=\frac{1}{\pi} \iint_{R \leq|z|<r} f^{\sharp}(z)^{2} d \sigma+O(1) \\
& \leq \frac{1}{\pi} \iint_{R \leq|z|<r} e^{\left.2|z|\right|^{\sigma(f)-\epsilon}} d \sigma+O(1)=\frac{1}{\pi} \int_{0}^{2 \pi} d \theta \int_{R}^{r} e^{2|z|^{\sigma(f)-\epsilon}} d t+O(1) \\
& \leq 2 r e^{2 r^{\sigma(f)-\epsilon}}[1+o(1)] .
\end{aligned}
$$

By the definition of Ahlfors characteristic of $f$, we have

$$
T(r, f)=\int_{0}^{r} \frac{S(t, f)}{t} d t \leq 2 r e^{2 r^{\sigma(f)-\epsilon}}[1+o(1)] .
$$

Then the hyper-order of $f$ is

$$
\sigma(f)=\limsup _{r \rightarrow \infty} \frac{\log \log T(r, f)}{\log r} \leq \limsup _{r \rightarrow \infty} \frac{(\sigma(f)-\epsilon) \log r}{\log r}=\sigma(f)-\epsilon,
$$

a contradiction. Thus, the proof is completed.

Lemma 2.3 [9] Let $f$ be a nonconstant entire function and let $k \geq 1$ be positive. Suppose that $f$ and $f^{(k)}$ share the value $1 C M$. Then $f-1$ has infinitely many zeros such that each 
zero off -1 is of order at most $k$, and

$$
N\left(r, \frac{1}{f-1}\right) \geq\left(\frac{1}{2}-\epsilon\right) T(r, f), \quad \bar{N}\left(r, \frac{1}{f-1}\right) \geq\left(\frac{1}{k+1}-\epsilon\right) T(r, f),
$$

for any $\epsilon>0$ and large enough $r$, and

$$
\delta(1, f) \leq \frac{1}{2}, \quad \Theta(1, f) \leq \frac{k}{1+k}
$$

Remark 4 There exist some mistakes in Theorem 2.2 and its proof and the proof of Theorem 3.1 in [9], we will correct them in another paper.

Lemma 2.4 [1] Let $f$ be a meromorphic function and $k \geq 1$ be positive. Then

$$
N\left(r, \frac{1}{f^{(k)}}\right) \leq N\left(r, \frac{1}{f}\right)+k \bar{N}(r, f)+S(r, f) .
$$

Lemma 2.5 [4] Let $f(z)$ be a meromorphic function in the complex plane, $\rho(f)>2$, then for each $0<\mu<\frac{\rho(f)-2}{2}$, there exist points $a_{n} \rightarrow \infty(n \rightarrow \infty)$ such that

$$
\lim _{n \rightarrow \infty} \frac{f^{\sharp}\left(a_{n}\right)}{\left|a_{n}\right|^{\mu}}=+\infty \text {. }
$$

Lemma 2.6 [10] Let $f(z)$ be an entire function in the complex plane, $\rho(f)>1$, then for each $0<\mu<\rho(f)-1$, there exist points $a_{n} \rightarrow \infty(n \rightarrow \infty)$ such that

$$
\lim _{n \rightarrow \infty} \frac{f^{\sharp}\left(a_{n}\right)}{\left|a_{n}\right|^{\mu}}=+\infty \text {. }
$$

\section{The proof of Theorem 1.1}

The proof of Theorem 1.1 In a similar way to that of $[8,11]$, we prove Theorem 1.1 as follows.

Noting that $f$ and $R$ have at most finitely many poles, there exists a positive number $r$ such that $f$ and $R$ have no poles in $D=\{z:|z| \geq r\}$. Then $f$ and $R$ are holomorphic in $D$.

Noting that $\alpha=\operatorname{Re}^{\gamma}$, we have $\sigma(\alpha)=\rho(\gamma)$, and then we just need to prove $\sigma(f) \leq \rho(\gamma)$.

On the contrary, suppose that $\sigma(f)=d>c=\rho(\gamma)$. Set $F=\frac{f}{\alpha}$, obviously, $\sigma(F)=\sigma(f)=d$. Then, for $0<\epsilon<\frac{d-c}{2}$, by Lemma 2.2, there exists a sequence $w_{n} \rightarrow \infty$ as $n \rightarrow \infty$ such that

$$
F^{\sharp}\left(w_{n}\right)>e^{\left|w_{n}\right|^{\sigma(F)-\epsilon}}=e^{\left|w_{n}\right|^{d-\epsilon}} .
$$

In view of $w_{n} \rightarrow \infty$ as $n \rightarrow \infty$, without loss of generality, we may assume that $\left|w_{n}\right| \geq r+1$ for all $n$. Define $D_{1}=\{z:|z|<1\}$ and

$$
F_{n}(z)=F\left(w_{n}+z\right)=\frac{f\left(w_{n}+z\right)}{\alpha\left(w_{n}+z\right)} \text {. }
$$

Then all $F_{n}(z)$ are holomorphic in $D_{1}$.

Thus, we structure a family of holomorphic functions $\left(F_{n}\right)_{n}$. Moreover,

$$
F_{n}^{\sharp}(0)=F^{\sharp}\left(w_{n}\right) \rightarrow \infty \quad \text { as } n \rightarrow \infty .
$$

It follows from Marty's criterion that $\left(F_{n}\right)_{n}$ is not normal at $z=0$. 
Therefore, applying Lemma 2.1 with $\alpha=k$ and choosing an appropriate subsequence of $\left(F_{n}\right)_{n}$ if necessary, we may assume that there exist sequences $\left(z_{n}\right)_{n} \in D_{1}$ and $\left(\rho_{n}\right)_{n}$ such that $\rho_{n} \rightarrow 0$ and

$$
g_{n}(\zeta)=\rho_{n}^{-k} F_{n}\left(z_{n}+\rho_{n} \zeta\right)=\rho_{n}^{-k} \frac{f\left(w_{n}+z_{n}+\rho_{n} \zeta\right)}{\alpha\left(w_{n}+z_{n}+\rho_{n} \zeta\right)} \rightarrow g(\zeta)
$$

locally uniformly in $\mathbb{C}$, where $g$ is a nonconstant entire function of order at most 1 , all zeros of $g$ have multiplicity at least $k+1$, and

$$
\rho_{n} \leq \frac{M}{F_{n}^{\sharp}(0)}=\frac{M}{F^{\sharp}\left(w_{n}\right)} \leq M e^{-\left|w_{n}\right|^{d-\epsilon}}
$$

for a positive number $M$.

We claim that

$$
\frac{f^{(l)}\left(w_{n}+z_{n}+\rho_{n} \zeta\right)}{\rho_{n}^{k-l} \alpha\left(w_{n}+z_{n}+\rho_{n} \zeta\right)} \rightarrow g^{(l)}(\zeta) \quad(0 \leq l \leq k)
$$

locally uniformly in $\mathbb{C}$. Obviously, the claim is correct if $l=0$. Next, we will prove that the claim holds by mathematical induction. We may assume that (3.4) holds for $l=s, 0 \leq s<k$, and then

$$
H_{n}(\zeta)=\frac{f^{(s)}\left(w_{n}+z_{n}+\rho_{n} \zeta\right)}{\rho_{n}^{k-s} \alpha\left(w_{n}+z_{n}+\rho_{n} \zeta\right)} \rightarrow g^{(s)}(\zeta)
$$

Now we will prove that (3.4) still holds for $l=s+1$. Differentiating (3.5), we deduce

$$
\frac{f^{(s+1)}\left(w_{n}+z_{n}+\rho_{n} \zeta\right)}{\rho_{n}^{k-s-1} \alpha\left(w_{n}+z_{n}+\rho_{n} \zeta\right)}=H_{n}^{\prime}(\zeta)+\frac{\rho_{n} H_{n}(\zeta) \alpha^{\prime}\left(w_{n}+z_{n}+\rho_{n} \zeta\right)}{\alpha\left(w_{n}+z_{n}+\rho_{n} \zeta\right)} .
$$

In view of the definition of order, we have

$$
\begin{aligned}
\left|\frac{\alpha^{\prime}}{\alpha}\right|_{z=w_{n}+z_{n}+\rho_{n} \zeta} \mid & =\left|\frac{R^{\prime}+R \gamma^{\prime}}{R}\right|_{z=w_{n}+z_{n}+\rho_{n} \zeta} \mid \\
& \leq\left|w_{n}\right|^{q} M\left(\left|w_{n}+z_{n}+\rho_{n} \zeta\right|, \gamma^{\prime}\right) \\
& \leq\left|w_{n}\right|^{q} M\left(2\left|w_{n}\right|, \gamma^{\prime}\right) \\
& \leq\left|w_{n}\right|^{q} e^{A\left|w_{n}\right|^{c+\epsilon}},
\end{aligned}
$$

where $A$ is a positive constant and $q$ is an integer.

Noting that $0<\epsilon<\frac{d-c}{2}$, we have $d-\epsilon>c+\epsilon$. Then combining (3.3) and (3.7) yields

$$
\begin{aligned}
\frac{\rho_{n} H_{n}(\zeta) \alpha^{\prime}\left(w_{n}+z_{n}+\rho_{n} \zeta\right)}{\alpha\left(w_{n}+z_{n}+\rho_{n} \zeta\right)} & =\frac{\rho_{n} H_{n}(\zeta)\left[R^{\prime}+R \gamma^{\prime}\right]\left(w_{n}+z_{n}+\rho_{n} \zeta\right)}{R\left(w_{n}+z_{n}+\rho_{n} \zeta\right)} \\
& \leq M\left|g_{n}(\zeta)\right|\left|w_{n}\right|^{q} e^{A\left|w_{n}\right|^{c+\epsilon}-\left|w_{n}\right|^{d-\epsilon}} \rightarrow 0
\end{aligned}
$$

as $n \rightarrow \infty$. By (3.6) and (3.8), we obtain

$$
\frac{f^{(s+1)}\left(w_{n}+z_{n}+\rho_{n} \zeta\right)}{\rho_{n}^{k-s-1} \alpha\left(w_{n}+z_{n}+\rho_{n} \zeta\right)} \rightarrow g^{(s+1)}(\zeta)
$$


So, (3.4) holds. Set $l=k$, then

$$
\frac{f^{(k)}\left(w_{n}+z_{n}+\rho_{n} \zeta\right)}{\alpha\left(w_{n}+z_{n}+\rho_{n} \zeta\right)} \rightarrow g^{(k)}(\zeta)
$$

Now, if $g^{(k)}(\zeta) \equiv 1$, then we obtain that $g(\zeta)$ is a polynomial of degree at most $k$. It contradicts with all zeros of $g$ having multiplicity at least $k+1$. Suppose that $g^{(k)}\left(\zeta_{0}\right)=1$, then by Hurwitz's theorem there exist $\zeta_{n}, \zeta_{n} \rightarrow \zeta_{0}$ such that (for sufficiently large $n$ )

$$
\frac{f^{(k)}\left(w_{n}+z_{n}+\rho_{n} \zeta\right)}{\alpha\left(w_{n}+z_{n}+\rho_{n} \zeta\right)}=1
$$

Noting that $f^{(k)}(z)=\alpha(z) \Rightarrow f(z)=\alpha(z)$ and (3.2), we obtain $g\left(\zeta_{0}\right)=\infty$. It contradicts with the assumption that $g^{(k)}\left(\zeta_{0}\right)=1$. So, $g^{(k)}(\zeta) \neq 1$.

Next, by the famous Hayman inequality for $g(\zeta)$, it is easy to obtain contradiction.

Hence, we complete the proof of Theorem 1.1.

\section{Proof of Theorem 1.2}

Let $H=f-Q_{1}$, then we have

$$
H=0 \quad \Rightarrow \quad H^{\prime}=Q_{1}-Q_{1}^{\prime}, \quad H=Q_{2}-Q_{1} \quad \Rightarrow \quad H^{\prime}=Q_{2}-Q_{1}^{\prime} .
$$

Let $P=Q_{2}-Q_{1}$ and $P_{2}=Q_{2}-Q_{1}^{\prime}$. Then $P \not \equiv 0$. We consider the function $F=\frac{H}{P}$, obviously, $\rho(F)=\rho(f)$

If $\rho(F)>2+2 \max \left\{\operatorname{deg}\left(\frac{Q_{2}-Q_{1}^{\prime}}{Q_{2}-Q_{1}}\right), \operatorname{deg}\left(\frac{Q_{1}-Q_{1}^{\prime}}{Q_{2}-Q_{1}}\right)\right\}$. By Lemma 2.5 , for each $0<\mu<\frac{\rho(f)-2}{2}$, there exist $w_{n} \rightarrow \infty$ such that for $n \rightarrow \infty$,

$$
\lim _{n \rightarrow \infty} \frac{F^{\sharp}\left(w_{n}\right)}{\left|w_{n}\right|^{\mu}}=+\infty \text {. }
$$

Since $P$ is a polynomial, we know that for any $\epsilon>0$, there exists an $r_{1}>0$ such that

$$
\left|\frac{P^{\prime}(z)}{P(z)}\right|<\epsilon \quad \text { and } \quad P(z) \neq 0
$$

for all $z \in \mathbb{C}$ satisfying $|z| \geq r_{1}$. Note that $f(z)$ has only finitely many poles, hence $H(z)$ has only finitely many poles. Thus, there is an $r_{2}>0$ such that $H(z)$ is holomorphic in $|z| \geq r_{2}$. Let $r=\max \left\{r_{1}, r_{2}\right\}$ and $D=\{z:|z| \geq r\}$, then $F$ is holomorphic in $D$. Without loss of generality, we may assume that $\left|w_{n}\right| \geq r+1$ for all $n$. We define $D_{1}=\{z:|z|<1\}$ and

$$
F_{n}(z)=F\left(w_{n}+z\right)
$$

Then all $F_{n}(z)$ are holomorphic in $D_{1}$ and $F_{n}^{\sharp}(0)=F^{\sharp}\left(w_{n}\right) \rightarrow \infty$ as $n \rightarrow \infty$. It follows from Marty's criterion that $\left(F_{n}\right)_{n}$ is not normal at $z=0$.

Therefore, by using Lemma 2.1 and choosing an appropriate subsequence of $\left(F_{n}\right)_{n}$ if necessary, we may assume that there exist sequences $\left(z_{n}\right)_{n}$ and $\left(\rho_{n}\right)_{n}$ with $\left|z_{n}\right|<r<1$ and $\rho_{n} \rightarrow 0$ such that the sequences $\left(g_{n}\right)_{n}$ are defined by

$$
g_{n}(\zeta)=F_{n}\left(z_{n}+\rho_{n} \zeta\right)=\frac{H\left(w_{n}+z_{n}+\rho_{n} \zeta\right)}{P\left(w_{n}+z_{n}+\rho_{n} \zeta\right)} \rightarrow g(\zeta)
$$


locally uniformly in $\mathbb{C}$ with a nonconstant entire function $g$ and

$$
\rho_{n} \leq \frac{M}{F_{n}^{\sharp}(0)}=\frac{M}{F^{\sharp}\left(w_{n}\right)}
$$

for a positive number $M$. Let $G_{n}(\zeta)=\rho_{n} \frac{H^{\prime}\left(w_{n}+z_{n}+\rho_{n} \zeta\right)}{P\left(w_{n}+z_{n}+\rho_{n} \zeta\right)}$, then from (4.2) and $\left|\frac{P^{\prime}\left(w_{n}+z_{n}+\rho_{n} \zeta\right)}{P\left(w_{n}+z_{n}+\rho_{n} \zeta\right)}\right|<1$ as $n \rightarrow \infty$, we get

$$
G_{n}(\zeta)=g_{n}^{\prime}(\zeta)+\frac{\rho_{n} g_{n}(\zeta) P^{\prime}\left(w_{n}+z_{n}+\rho_{n} \zeta\right)}{P\left(w_{n}+z_{n}+\rho_{n} \zeta\right)} \rightarrow g^{\prime}(\zeta)
$$

locally uniformly in $\mathbb{C}$.

Suppose that $g\left(\zeta_{0}\right)=1$, then by Hurwitz's theorem there exist $\zeta_{n}, \zeta_{n} \rightarrow \zeta_{0}$ such that (for $n$ sufficiently large)

$$
g_{n}\left(\zeta_{n}\right)=\frac{H\left(w_{n}+z_{n}+\rho_{n} \zeta_{n}\right)}{P\left(w_{n}+z_{n}+\rho_{n} \zeta_{n}\right)}=1
$$

By the assumption of Theorem 1.2, we have

$$
H^{\prime}\left(w_{n}+z_{n}+\rho_{n} \zeta_{n}\right)=P_{2}\left(w_{n}+z_{n}+\rho_{n} \zeta_{n}\right)
$$

From (4.1) and (4.3), we deduce that

$$
\lim _{n \rightarrow \infty} w_{n}^{l_{1}} \rho_{n}=0
$$

for any fixed constant $0 \leq l_{1}<\frac{\rho(f)-2}{2}$. Meanwhile, we have

$$
\frac{P_{2}\left(w_{n}+z_{n}+\rho_{n} \zeta\right)}{P\left(w_{n}+z_{n}+\rho_{n} \zeta\right)}=O\left(\left|w_{n}\right|^{l_{1}}\right)
$$

here $\operatorname{deg} R_{1}=\operatorname{deg}\left(\frac{P_{2}}{P}\right)=\operatorname{deg}\left(\frac{Q_{2}-Q_{1}^{\prime}}{Q_{2}-Q_{1}}\right)=l_{1}$. By (4.6), we deduce that

$$
\rho_{n} \frac{P_{2}\left(w_{n}+z_{n}+\rho_{n} \zeta\right)}{P\left(w_{n}+z_{n}+\rho_{n} \zeta\right)}=O\left(\rho_{n} w_{n}^{l_{1}}\right) \rightarrow 0 \quad(n \rightarrow \infty) .
$$

By (4.4), (4.5) and (4.7), we obtain that

$$
g^{\prime}\left(\zeta_{0}\right)=\lim _{n \rightarrow \infty} \rho_{n} \frac{H^{\prime}\left(w_{n}+z_{n}+\rho_{n} \zeta_{n}\right)}{P\left(w_{n}+z_{n}+\rho_{n} \zeta_{n}\right)}=\lim _{n \rightarrow \infty} \rho_{n} \frac{P_{2}\left(w_{n}+z_{n}+\rho_{n} \zeta_{n}\right)}{P\left(w_{n}+z_{n}+\rho_{n} \zeta_{n}\right)}=0 .
$$

Thus $g(\zeta)=1 \Rightarrow g^{\prime}(\zeta)=0$, which yields that the zeros of $g-1$ are of multiplicity at least 2 . Similarly, we can prove that the zeros of $g$ are of multiplicity at least 2 .

Noting that $Q_{1}(z) \not \equiv Q_{2}(z)$, without loss of generality, we assume that $Q_{1}(z) \not \equiv 0$. Next, we shall prove that $g(\zeta) \neq 0$. Suppose that $\xi_{0}$ is a zero of $g(\zeta)$ with multiplicity $m(\geq 2)$, then $g^{(m)}\left(\xi_{0}\right) \neq 0$. Thus there exists a positive number $\delta$ such that

$$
g(\zeta) \neq 0, \quad g^{\prime}(\zeta) \neq 0, \quad g^{(m)}(\zeta) \neq 0
$$

on $D_{\delta}^{o}=\left\{z: 0<\left|\zeta-\xi_{0}\right|<\delta\right\}$. 
Noting that $g(\zeta) \not \equiv 0$, by Rouché's theorem there exist $\zeta_{n, j}(j=1,2, \ldots, m)$ on $D_{\delta / 2}=\{\xi$ : $\left.\left|\zeta-\xi_{0}\right|<\delta / 2\right\}$ such that

$$
g_{n}\left(\zeta_{n, j}\right)=H\left(w_{n}+z_{n}+\rho_{n} \zeta_{n, j}\right)=0 \quad(j=1, \ldots, m)
$$

Note that, for $n$ large enough,

$$
H^{\prime}\left(w_{n}+z_{n}+\rho_{n} \zeta_{n, j}\right)=Q_{1}\left(w_{n}+z_{n}+\rho_{n} \zeta_{n, j}\right)-Q_{1}^{\prime}\left(w_{n}+z_{n}+\rho_{n} \zeta_{n, j}\right) \neq 0
$$

so each $\zeta_{n, j}$ is a simple zero of $H\left(w_{n}+z_{n}+\rho_{n} \zeta\right)$, that is, $\zeta_{n, j} \neq \zeta_{n, i}(1 \leq i \neq j \leq m)$.

Likewise (4.7), we have

$$
\rho_{n} \frac{Q_{1}\left(w_{n}+z_{n}+\rho_{n} \zeta\right)-Q_{1}^{\prime}\left(w_{n}+z_{n}+\rho_{n} \zeta\right)}{P\left(w_{n}+z_{n}+\rho_{n} \zeta\right)}=O\left(\rho_{n} w_{n}^{l_{2}}\right) \rightarrow 0 \quad(n \rightarrow \infty)
$$

here $l_{2}=\operatorname{deg} R_{2}=\operatorname{deg} \frac{Q_{1}-Q_{1}^{\prime}}{Q_{2}-Q_{1}}$.

Note that by (4.4) and (4.9) we have

$$
K_{n}(\zeta)=G_{n}(\zeta)-\rho_{n} \frac{Q_{1}\left(w_{n}+z_{n}+\rho_{n} \zeta\right)-Q_{1}^{\prime}\left(w_{n}+z_{n}+\rho_{n} \zeta\right)}{P\left(w_{n}+z_{n}+\rho_{n} \zeta\right)} \rightarrow g^{\prime}(\zeta)
$$

and $K_{n}\left(\zeta_{n, j}\right)=0(j=1, \ldots, m)$. From $(4.8)$ we have

$$
\lim _{n \rightarrow \infty} \zeta_{n, j}=\xi_{0} \quad(j=1,2, \ldots, m)
$$

Noting (4.8), (4.10) and that $K_{n}(\zeta)$ has $m$ zeros $\zeta_{n, j}(j=1,2, \ldots, m)$ in $D_{\delta / 2}$, we obtain from Hurwitz's theorem that $\xi_{0}$ is a zero of $g^{\prime}(\zeta)$ with multiplicity $m$, and thus $g^{(m)}\left(\xi_{0}\right)=0$. This is a contradiction. Hence $g(\zeta) \neq 0$.

We have shown that $g$ is a nonvanishing entire function that takes the value 1 always with multiplicity at least 2 . But this contradicts Nevanlinna's second fundamental theorem that the sum of the defects is at most 2 .

This completes the proof of Theorem 1.2.

\section{The proof of Corollary $\mathbf{1 . 3}$}

By Lemma 2.6, the reader could give the proof of Corollary 1.3 with almost the same argument as that in the proof of Theorem 1.2. Here we omit it.

\section{The proof of Theorem 1.4}

By a similar way to that of $\mathrm{Li}, \mathrm{Gao}$ and Zhang [9], we prove Theorem 1.4 as follows.

Since $f$ is an entire function, by Lemma 2.4 we have

$$
N\left(r, \frac{1}{f^{(k+1)}}\right) \leq N\left(r, \frac{1}{f^{(k)}}\right)+S(r, f)<\alpha T(r, f)+S(r, f) .
$$

Set

$$
F=\frac{f^{(k+2)}}{f^{(k+1)}}-\frac{f^{(k+1)}}{f^{(k)}}-2 \frac{f^{(k+1)}}{f^{(k)}-1}+2 \frac{f^{\prime}}{f-1} .
$$


Then $F$ is a meromorphic function and hence

$$
m(r, F)=S(r, f)
$$

from the fundamental estimate of the logarithmic derivative. Since the poles of $F$ appear to the zeros of $f^{(k)}$ and $f^{(k+1)}$, by assumption and (6.1), we have

$$
N(r, F) \leq N\left(r, \frac{1}{f^{(k)}}\right)+N\left(r, \frac{1}{f^{(k+1)}}\right)+S(r, f) .
$$

Combining (6.3) with (6.4), we see that $T(r, F)<2 \alpha T(r, f)+S(r, f)$.

We assume that $F \not \equiv 0$. Notice that all zeros of $f-1$ and $f^{(k)}-1$ are $k$ multiples. Let $z_{0}$ be a common zero of $f-1$ and $f^{(k)}-1$. Then $f^{(k+1)}\left(z_{0}\right) \neq 0$, and it is easy to see that $F$ is holomorphic at $z_{0}$, and $F\left(z_{0}\right)=0$. Thus we have

$$
\begin{aligned}
N\left(r, \frac{1}{f-1}\right) & \leq N\left(r, \frac{1}{F}\right) \leq T(r, F)+O(1) \\
& \leq 2 \alpha T(r, f)+S(r, f)<2\left(\alpha+\frac{1-4 \alpha}{8}\right) T(r, f) \\
& =\left(\alpha+\frac{1}{4}\right) T(r, f),
\end{aligned}
$$

for large enough $r$. However, by Lemma 2.3, we have

$$
N\left(r, \frac{1}{f-1}\right) \geq\left(\frac{1}{2}-\left(\frac{1}{4}-\alpha\right)\right) T(r, f)=\left(\alpha+\frac{1}{4}\right) T(r, f),
$$

for large enough $r$. (6.6) contradicts to (6.5).

Hence $F \equiv 0$. Integration of (6.2) yields

$$
A \frac{f^{(k+1)}}{f^{(k)}}=\left(\frac{f^{(k)}-1}{f-1}\right)^{2}
$$

where $A$ is a nonzero constant. Since $f$ and $f^{(k)}$ share $1 \mathrm{CM}$, for any point $z_{1}$ satisfying that $f\left(z_{1}\right)=f^{(k)}\left(z_{1}\right)=1$, we obtain that $f^{\prime}\left(z_{1}\right) f^{(k+1)}\left(z_{1}\right) \neq 0$, and then $A=\frac{f^{(k+1)}\left(z_{1}\right)}{\left[f^{\prime}\left(z_{1}\right)\right]^{2}}$. Thus if we assume that $\frac{f^{(k+1)}}{f^{(k)}}$ is not a constant function, we see from (6.7) that $f^{(k)}(z) \neq 0$ and $f^{(k+1)}(z) \neq 0$. Noting that $f(z)$ is an entire function, we see that $\frac{f^{(k+1)}}{f^{(k)}}$ has no both pole and zero. So $A \frac{f^{(k+1)}}{f^{(k)}}=: e^{h(z)}$ is a small function of $f^{(k)}$, where $h(z)$ is an entire function. Now we may change (6.7) into

$$
f(z)=\left(f^{(k)}(z)-1\right) e^{-\frac{1}{2} h(z)}+1 .
$$

By the second main theorem, (6.5) and (6.8), we have

$$
\begin{aligned}
T(r, f) & =T\left(r, f^{(k)}\right)+S\left(r, f^{(k)}\right) \\
& \leq N\left(r, f^{(k)}\right)+N\left(r, \frac{1}{f^{(k)}}\right)+N\left(r, \frac{1}{f^{(k)}-1}\right)+S\left(r, f^{(k)}\right)
\end{aligned}
$$




$$
\begin{aligned}
& \leq N\left(r, \frac{1}{f-1}\right)+S(r, f) \\
& \leq \frac{1}{2} T(r, f)+S(r, f)
\end{aligned}
$$

a contradiction.

Therefore, $\frac{f^{(k+1)}}{f^{(k)}}$ is a constant function and hence there exists a nonzero constant $c$ such that

$$
\frac{f^{(k+1)}(z)-1}{f(z)-1}=c .
$$

\section{This completes the proof of Theorem 1.4.}

\section{Competing interests}

The authors declare that they have no competing interests.

\section{Authors' contributions}

JQ carried out the main part of this manuscript. FL and WY participated discussion and corrected the main theorem. All authors read and approved the final manuscript.

\section{Author details}

'Department of Mathematics and Physics, Shanghai Dianji University, Shanghai, 201306, P.R. China. ${ }^{2}$ College of Science, China University of Petroleum, Qingdao, 266555, P.R. China. ${ }^{3}$ School of Mathematics and Information Science, Guangzhou University, Guangzhou, 510006, P.R. China. ${ }^{4}$ Key Laboratory of Mathematics and Interdisciplinary Sciences of Guangdong Higher Education Institutes, Guangzhou University, Guangzhou, 510006, P.R. China.

\section{Acknowledgements}

This work was supported by the Visiting Scholar Program of Chern Institute of Mathematics at Nankai University when the authors worked as visiting scholars. The authors would like to express their hearty thanks to Chern Institute of Mathematics that provided very comfortable research environment to them. Project supported by the funding scheme for training young teachers in colleges and universities in Shanghai (ZZSDJ12020), also supported by the NNSF of China (No. 11271090, 11171184, 11001057), the NSF of Guangdong Province (S2012010010121) and by project 10XKJ01, 12C401 and 12C104 from the Leading Academic Discipline Project of Shanghai Dianji University. The authors wish to thank the referee and managing editor for their very helpful comments and useful suggestions.

Received: 7 January 2013 Accepted: 29 May 2013 Published: 1 July 2013

\section{References}

1. Yi, HX, Yang, CC: Uniqueness Theory of Meromorphic Functions. Science Press, Beijing (1995)

2. Rubel, LA, Yang, CC: Values shared by an entire function and its derivative. In: Complex Analysis, pp. 101-103. Lecture Notes in Math., vol. 599. Springer, Berlin (1976)

3. Liu, XJ, Nevo, S, Pang, XC: On the kth derivative of meromorphic functions with zeros of multiplicity at least $k+1$. J. Math. Anal. Appl. 348, 516-529 (2008)

4. Yuan, WJ, Xiao, B, Zhang, JJ: The general result of Gol'dberg's theorem concerning the growth of meromorphic solutions of algebraic differential equations. Comput. Math. Appl. 58, 1788-1791 (2009)

5. Bank, S, Laine, I: On the oscillation theory of $f^{\prime \prime}+A(z) f=0$ where $A$ is entire. Trans. Am. Math. Soc. 273, 351-363 (1982)

6. Li, XM, Gao, CC: Entire functions sharing one polynomial with their derivatives. Proc. Indian Acad. Sci. Math. Sci. 118, 13-26 (2008)

7. Lü, F: A note on the Brük conjecture. Bull. Korean Math. Soc. 48, 951-957 (2011)

8. Lü, F, Xu, JF, Chen, A: Entire functions sharing polynomials with their first derivatives. Arch. Math. 92, 593-601 (2009)

9. Li, S, Gao, ZS, Zhang, JL: Entire functions that share values or small functions with their derivatives. Ann. Pol. Math. 104, 1-11 (2012)

10. Gu, RM, Li, ZR, Yuan, WJ: The growth of entire solutions of some algebraic differential equations. Georgian Math. J. 18 489-495 (2011)

11. Grahl, J, Meng, C: Entire functions sharing a polynomial with their derivatives and normal families. Analysis 28, 51-61 (2008) 